\title{
11. Trinitrobenzene Sulfonic Acid (TNBS) Increases Transmitter Release at the Frog Neuromuscular Junction
}

\author{
By Noriko Tanabe, ${ }^{*}$ Hiromasa KiJima, ${ }^{* *}$ and Juro Maruhashi*) \\ (Communicated by Yasuji Katsuki, M. J. A., Jan. 12, 1987)
}

Introduction. Presynaptic processes of vertebrate neuromuscular transmission consist of nerve terminal membrane depolarization by action potentials, inflow of calcium ion through voltage gated channels into the presynaptic terminal and the transmitter release into the synaptic cleft.12) However, the processes underlying the relationship between calcium influx and the transmitter release still remain obscure. Many agents, such as lithium ion, ${ }^{6)}$ calcium ionophore, ${ }^{14), 18}$ ) erythrosin $\mathrm{B}^{3)}$ and metabolic or sodium pump inhibitors ${ }^{7), 9)}$ have been known to augment both the amplitude of nerve evoked endplate potentials (epps) and the frequency of spontaneous miniature endplate potentials (mepps). All these agents are thought to act by elevating the calcium concentrations within the nerve terminal. Some sulfhydryl and disulfide reagents were also reported to increase mepp frequency.5),17),19) Here we report an amino group modifying reagent, 2,4,6-trinitrobenzene 1 -sulfonic acid $(\mathrm{TNBS}),{ }^{15)}$ rapidly increases both the epp amplitudes and mepp frequencies.

Materials and methods. Cutaneous pectoris and sartorius nerve muscle preparations isolated from the frogs, Rana nigromaculata and $R$. catesbeiana were used. Membrane potential changes were recorded intracellularly at endplate regions with a glass micro-electrode filled with $3 \mathrm{M} \mathrm{KCl}$. All data were fed to a PCM data recorder, A/D converted, and processed by a microcomputer. Drugs were applied by perfusion. The high magnesium Ringer solution containing $\mathrm{NaCl}(112 \mathrm{mM}), \mathrm{KCl}(2.6 \mathrm{mM}), \mathrm{CaCl}_{2}(2.4 \mathrm{mM}), \mathrm{MgCl}_{2}(20 \mathrm{mM})$ and HEPES $(5 \mathrm{mM}, \mathrm{pH} 7.5)$ were used unless otherwise noted. The epp was evoked by stimulating a nerve with a suction electrode every $2 \mathrm{sec}$. The quantal content and quantal size of epps were analyzed by the variance method.10) All experiments were performed at room temperature $\left(21-23^{\circ} \mathrm{C}\right)$.

Results. Application of $2 \mathrm{mM}$ TNBS to the frog endplate increased the peak amplitude of epp. Augmentation of epp reached its maximum 3-7 min after onset of TNBS application (3.1 \pm 0.9 times larger than the control, $n=13)$ and usually decreased gradually with time as shown in Fig. 1. During TNBS application up to $40 \mathrm{~min}$, the quantal size was kept unchanged but only quantal content varied with the change of epp amplitude. To examine the postsynaptic effect of TNBS, carbamyl choline ( $\mathrm{CCh}$ ) was applied iontophoretically to an endplate region. Induced postsynapstic potentials were almost unchanged during TNBS application up to $60 \mathrm{~min}$, indicating that postsynaptic membrane was little affected by TNBS (not shown in the figure).

\footnotetext{
*) Department of Biology, Daiichi College of Pharmaceutical Sciences, Fukuoka 815, Japan.

**) Department of Biology, Faculty of Science, Kyushu University, Fukuoka 812, Japan.
} 


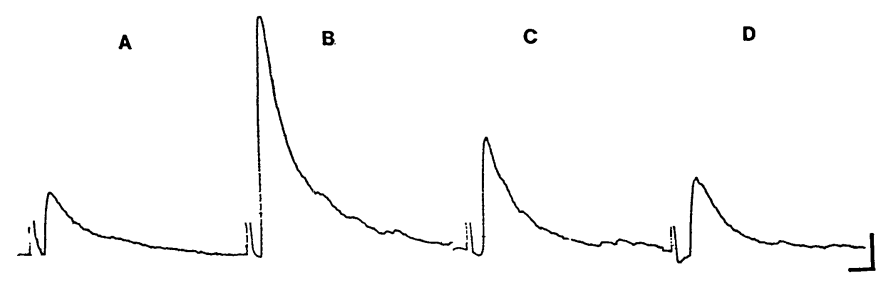

Fig. 1. Increase in epp amplitudes by TNBS treatment at an endplate. A : control. B : 7 min after. C: 25 min after. D: 60 min after the onset of TNBS treatment. The epp was maximum at (B). Resting membrane potential remained unchanged during the experiment $(-90 \mathrm{mV})$. Calibrations: $5 \mathrm{msec}$ and $1 \mathrm{mV}$.

In addition, TNBS caused enormous increase of spontaneous mepp frequency within several minutes after the onset of application and remained constant after about $10 \mathrm{~min}$ as shown in Fig. 2B. However, the distribution of mepp amplitude was unchanged, in agreement with the invariance of quantal size of epp. When the perfusion medium was changed to the normal solution after 20 min soaking in TNBS, mepp frequency decreased partially within ten min to the level much higher than the control level (Fig. 2A, B). Rapid appearance of TNBS effect and its partial but rapid reversal by washing show that it acted first from the outside of the presynaptic membrane. Even when the calcium ion was removed from the bath solution and a calcium chelator, EGTA $(1.2-3.6 \mathrm{mM})$ or EDTA $(1.8 \mathrm{mM})$, was added, TNBS increased mepp frequency in the same degree (Fig. 2C), although the control value before TNBS application was lower and the rate of increase was slower. Moreover, when the endplate was treated with $0.2 \mu \mathrm{M}$ synthetic $\omega$-conotoxin $(\omega$-CTX), a specific and potent calcium channel blocker,1),2),8),11),13),16),20) for $90 \mathrm{~min}$, the nerve evoked epp was completely blocked irreversibly but application of TNBS after epp block caused the same amount of increase in the mepp frequency as in the case of calcium removal (Fig. 2D). Both show that increase of mepp frequency by TNBS treatment was not caused by the modification of calcium influx.

The conclusion is that TNBS acts on the presynaptic membrane from the outside at least in the first step and augments both epp amplitude and mepp frequency by affecting other processes than the calcium influx.

Discussion. The accelerating effect of TNBS on both epp and mepp occurred very rapidly and drastically: It became appreciable within one minute after onset of TNBS perfusion. There are two possibilities remaining for further study. One is that TNBS may increase internal concentration of calcium ion by blocking its sequestering process or by inducing its internal mobilization and the other is that it may modify exocytotic processes of synaptic vesicles by affecting components in the presynaptic membrane.

TNBS was reported impermeable to the nerve membrane and thus it reacted from the outside.4) This is in agreement with our results that TNBS interacts first partially reversibly with some components on the outside of the presynaptic membrane. Similar mechanism was suggested on the action of sodium bisulfite. ${ }^{19)}$ The effect of mepp frequency became gradually irreversible (not shown in the figure), presumably due to its reaction with amino- or sulfhydryl groups of the protein components on the membrane. The epp was first augmented and gradually depressed by TNBS. These two phases of the effect on epp may correspond to 
A

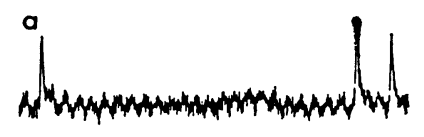

b

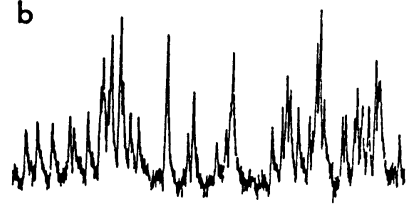

c

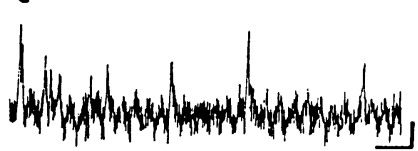

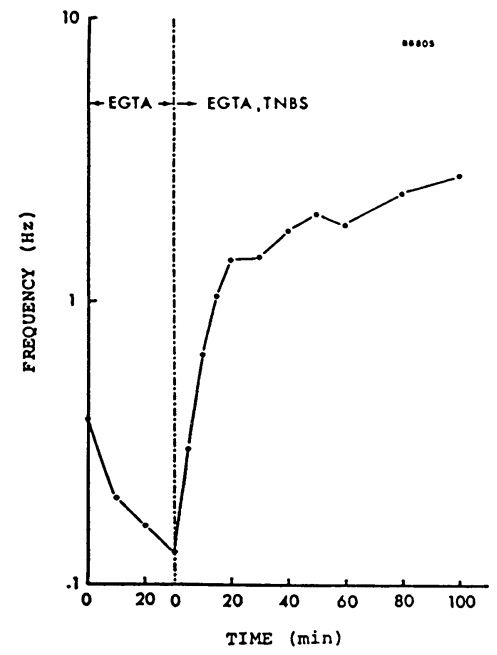

B

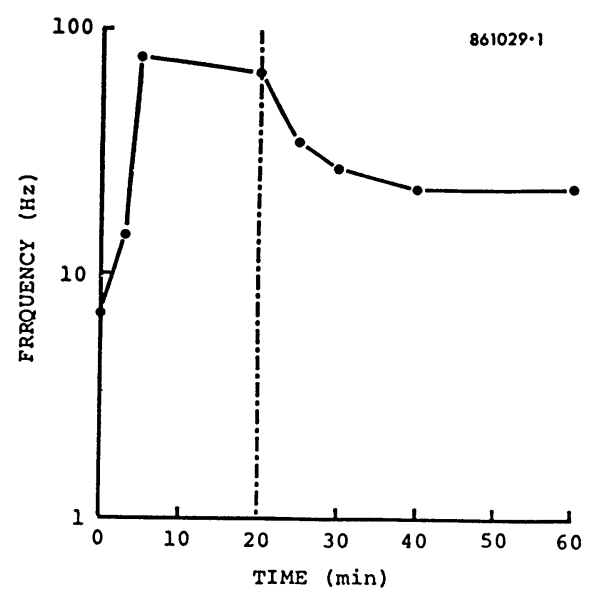

D

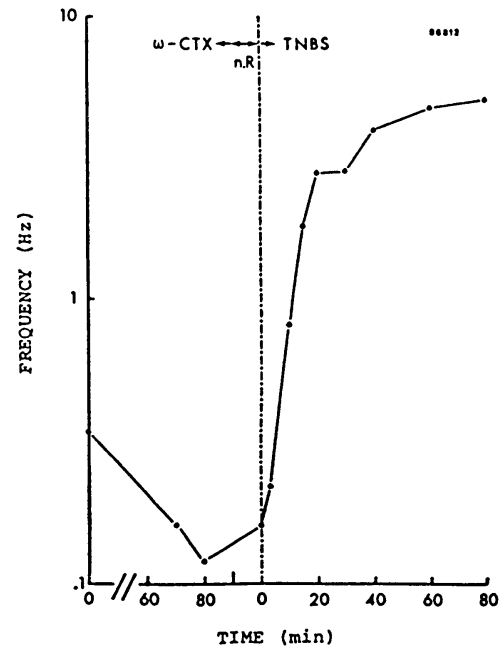

Fig. 2. Increase in mepp frequency. A: The mepp records of control(a), 5 min after the onset of TNBS treatment (b) and 10 min after TNBS was washed out (c) ; cf. (B). Calibrations: $50 \mathrm{msec}$ and $0.1 \mathrm{mV}$. B-D: Time course of the effect of TNBS on mepp frequency. Ordinate: mepp frequency calculated from the record for $1 \mathrm{~min}$ before and after the point (log scale). Abscissa: time (min) after the onset of TNBS perfusion. B: In high $\mathrm{Mg}^{2+}$ Ringer solution (the same experiment as in A). After treatment for $20 \mathrm{~min}$, TNBS was washed out by perfusing drug-free solution. $\mathrm{C}$ : In $\mathrm{Ca}^{2+}$-free and low $\mathrm{Mg}^{2+}(1.8 \mathrm{mM})$ Ringer solution containing $2 \mathrm{mM}$ EGTA. D: After treatment with $\omega$-CTX for $90 \mathrm{~min}$, $\omega$-CTX was washed out by low $\mathrm{Mg}^{2+}(2.0 \mathrm{mM})$ Ringer solution $\left(\mathrm{Ca}^{2+}: 0.9 \mathrm{mM}\right)$ and then TNBS was perfused. 
the reversible and the irreversible effects on mepps, but not yet ascertained. The fact that a protein modifying reagent, TNBS enormously affected the presynaptic processes should be useful for the elucidation of molecular processes of transmitter release.

Acknowledgments. The authors would like to thank Prof. T. Maeno, Dr. H. Kita and Dr. K. Enomoto for reading the manuscript and valuable comments and advice. This work is supported in part by a Grant-in-Aid (61107005) from the Japanese Ministry of Education, Science and Culture. The synthetic $\omega$-CTX was developed by the collaboration between Peptide Institute, Protein Research Foundation and the organizing group of Special Project Research on Mechanism of Bioelectrical Response, which was supported by a Grant-in-Aid (61107004) from the Japanese Ministry of Education, Science and Culture.

\section{References}

1) Abe, T. et al. (1986): Neurosci. Lett., 71, 203-208.

2) Abe, T., and Saisu, H. (1986) : Proc. Japan Acad., 62B, 416-418.

3) Augustin, G. J., and Levitan, H. (1983) : J. Physiol., 334, 47-63.

4) Cahalan, M. D., and Pappone, P. A. (1981): ibid., 321, 127-139.

5) Carmody, J. J. (1978) : European J. Pharmacol., 47, 457-460.

6) Crawford, A. C. (1975): J. Physiol., 246, 109-142.

7) Elmqvist, D., and Feldman, D. S. (1965) : ibid., 181, 498-505.

8) Enomoto, K. et al. (1986): Proc. Japan Acad., 62B, 267-270.

9) Glagoleva, I. M. et al. (1970): Biophysics, 15, 74-82.

10) Hubbard, J. I., Lilinas, R., and Quastel, D. M. J. (1969) : Electrophysiological Analysis of Synaptic Transmission. Edward Arnold, London, pp. 133-137.

11) Kasai, H., Aosaki, T., and Fukuda, J. (1986): Neurosci. Res. (in press).

12) Katz, B. (1969): The Release of Neural Transmitter Substances. Charles C. Thomas, Springfield.

13) Kerr, L. M., and Yoshikami D. (1984): Nature, 308, 282-284.

14) Kita, H., and Van der Kloot, W. (1976) : J. Physiol., 259, 177-198.

15) Means, G. E., and Feeney, R. E. (1971): Chemical Modification of Proteins. Holden-Day, Inc., San Francisco.

16) Mochida, S., and Kobayashi, H. (1986) : Neurosci. Lett., 72, 205-210.

17) Publicover, S. J., and Duncan, C. J. (1981) : European J. Pharmacol., 70, 203-211.

18) Statham, H. E., and Duncan, C. J. (1976) : Life Sci., 17, 1401-1406.

19) Steinacker, A. (1982) : J. Neurosci. Res., 7, 313-319.

20) Suzuki, N., and Yoshioka, T. (1986): Neurosci. Lett. (in press). 